\title{
Effectiveness of nivolumab versus regorafenib in hepatocellular carcinoma patients who failed sorafenib treatment
}

Cheol-Hyung Lee ${ }^{1, *}$, Yun Bin Lee ${ }^{1, *}$, Minseok Albert Kim ${ }^{1}$, Heejoon Jang', Hyunwoo Oh', Sun Woong Kim', Eun Ju Cho', Kyung-Hun Lee ${ }^{2,3}$, Jeong-Hoon Lee', Su Jong Yu', Jung-Hwan Yoon', Tae-You Kim ${ }^{2,3}$, and Yoon Jun Kim

${ }^{1}$ Department of Internal Medicine and Liver Research Institute, Seoul National University College of Medicine, Seoul; ${ }^{2}$ Department of Internal Medicine, Seoul National University Hospital, Seoul; ${ }^{3}$ Cancer Research Institute, Seoul National University, Seoul, Korea

\section{Graphical Abstract}

Kaplan-Meier curves of overall survival according to the treatment group:

Nivolumab may be associated with improved overall survival compared to regorafenib in HCC patients after sorafenib failure.

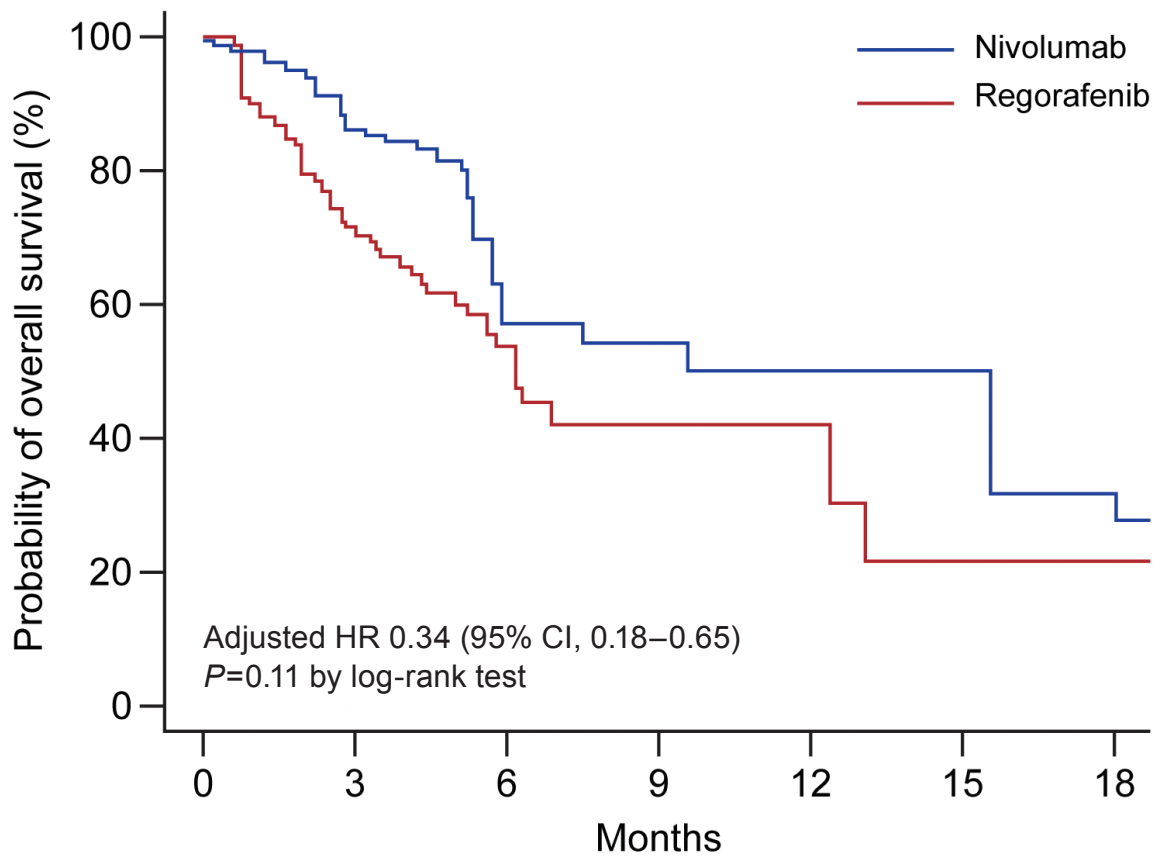

\section{Abbreviations:}

AFP, alpha-fetoprotein; aHR, adjusted hazard ratio; Cl, confidence interval; HCC, hepatocellular carcinoma; IPTW, inverse probability treatment weighting; IQR, interquartile range; mRECIST, modified Response Evaluation Criteria in Solid Tumors; NE, not estimable; OS, overall survival; RECIST, Response Evaluation Criteria in Solid Tumors; TTP, time to progression

* These authors contributed equally to this work.

\section{Corresponding author : Yoon Jun Kim}

Department of Internal Medicine and Liver Research Institute, Seoul National University College of Medicine, 101 Daehak-ro, Jongno-gu, Seoul 03080, Korea

Tel: +82-2-2072-2228, Fax: +82-2-743-6701

E-mail:yoonjun@snu.ac.kr

https://orcid.org/0000-0001-9141-7773 
Cheol-Hyung Lee, et al. Nivolumab versus regorafenib for advanced HCC

Background/Aims: Several treatment options are currently available for patients with hepatocellular carcinoma (HCC) failing previous sorafenib treatment. We aimed to compare the effectiveness of regorafenib and nivolumab in these patients.

Methods: Consecutive HCC patients who received regorafenib or nivolumab after failure of sorafenib treatment were included. Primary endpoint was overall survival (OS) and secondary endpoints were time to progression, tumor response rate, and adverse events. Inverse probability of treatment weighting (IPTW) using the propensity score was conducted to reduce treatment selection bias.

Results: Among 150 study patients, 102 patients received regorafenib and 48 patients received nivolumab. Median OS was 6.9 (95\% confidence interval [Cl], 3.0-10.8) months for regorafenib and $5.9(95 \% \mathrm{Cl}, 3.7-8.1)$ months for nivolumab ( $P=0.77$ by log-rank test). In multivariable analysis, nivolumab was associated with prolonged OS (vs. regorafenib: adjusted hazard ratio [aHR], $0.54 ; 95 \% \mathrm{Cl}, 0.30-0.96 ; P=0.04$ ). Time to progression was not significantly different between groups (nivolumab vs. regorafenib: aHR, $0.82 ; 95 \% \mathrm{Cl}, 0.51-1.30 ; P=0.48$ ). HRs were maintained after IPTW. Objective response rates were $5.9 \%$ and $16.7 \%$ in patients treated with regorafenib and nivolumab, respectively $(P=0.04)$.

Conclusions: After sorafenib failure, the use of nivolumab may be associated with improved OS and better objective response rate as compared to using regorafenib. (Clin Mol Hepatol 2020;26:328-339)

Keywords: Liver cancer; Regorafenib; Nivolumab; Survival

\section{Study Highlights}

With advances in systemic therapy for HCC, several treatments are now available for patients who experienced sorafenib treatment failure. This is the first study that compared the effectiveness of regorafenib and nivolumab in those patients. Nivolumab was associated with improved overall survival with a better objective response rate compared to regorafenib. The safety profiles were similar between the treatments, even though the patients treated with nivolumab were more likely to be with advanced liver disease compared to patients treated with regorafenib at baseline.

\section{INTRODUCTION}

Hepatocellular carcinoma (HCC) is estimated to be the sixth most commonly diagnosed cancer and the fourth major cause of cancer-related mortality worldwide, with 841,000 new cases and 782,000 deaths annually. ${ }^{1}$ Despite the efforts to improve public health, the age-standardized incidence rate is increasing in countries with a high socio-demographic index. ${ }^{2}$

Although sorafenib has been shown to improve overall survival (OS) of patients with advanced HCC, the prolongation of survival is modest. ${ }^{3}$ Recently, several therapeutic agents have been tested in patients with $\mathrm{HCC}$ who have progressed during sorafenib treatment or who were intolerant to sorafenib. ${ }^{4-8}$ In a placebo-controlled phase 3 study (RESORCE trial) in patients who had disease progression after sorafenib treatment, regorafenib, an oral multikinase inhibitor, showed improved OS. ${ }^{5}$ Median OS was 10.7 months for regorafenib and 7.9 months for placebo. ${ }^{9}$ In a non-comparative phase 2 study with nivolumab (CheckMate 040 trial), ${ }^{6}$ which is a human anti-programmed cell death-1 monoclonal antibody that disrupts immune checkpoint signaling, a sub- group analysis in patients who had previously treated with sorafenib demonstrated an objective response rate of $14 \%$ and disease control rate of $55 \%{ }^{10}$ Moreover, the median duration of response was 19.4 months, ${ }^{10}$ suggesting that nivolumab might offer durable tumor responses. Based on these promising results, nivolumab is currently available in several countries, including the United States and Korea, for the treatment of advanced HCC after sorafenib failure.

Though regorafenib and nivolumab have been proven effective in pivotal clinical trials, study results in real-world clinical practice are limited. ${ }^{11-15}$ In addition, a direct comparison of the efficacy of regorafenib and nivolumab has not been conducted to date. As sorafenib failed patients raised importance in clinical practice, we also included sorafenib intolerant patients. In this study, we aimed to evaluate and compare the effectiveness of regorafenib versus nivolumab in patients with HCC who had failed sorafenib treatment. 


\section{PATIENTS AND METHODS}

\section{Study population}

In this retrospective cohort study, we included consecutive HCC patients who had experienced disease progression or intolerance during sorafenib treatment and received regorafenib or nivolumab thereafter between July 1, 2015 and October 31, 2018 at Seoul National University Hospital (Seoul, Korea) (Fig. 1). The study conformed to the ethical guidelines of the World Medical Association Declaration of Helsinki and was approved by the Institutional Review Board of Seoul National University Hospital. The requirement to obtain informed consent was waived because existing administrative and clinical data were obtained from the electronic databases and analyzed retrospectively.

We included patients who met all of the following inclusion criteria: 1) were at least 18 years old; 2 ) had received regorafenib or nivolumab treatment after sorafenib failure; and 3) HCC was radiologically or histologically confirmed according to American Association for the Study of Liver Disease criteria, with measurable disease based on the Response Evaluation Criteria in Solid Tumors (RECIST; version 1.1). Patients were excluded from the study if any of following exclusion criteria met: 1) received prior immune-targeted therapy; 2) were participating in clinical trials; 3) prior liver transplantation; 4) unclear history of sorafenib treatment; and 5) had a concurrent malignancy other than HCC.

\section{Treatment}

The clinician comprehensively assessed the clinical situation of the patient, including side effects during the previous sorafenib treatment and residual liver function to select the subsequent treatment after sorafenib failure. The initial dose of regorafenib was $160 \mathrm{mg}$ once daily for the first 21 days of each 28-day cycle. Regorafenib dose adjustment by the amount of $40 \mathrm{mg}$ or transient interruption (<14 days) owing to drug-related toxicity was allowed. Nivolumab was administered intravenously every 2 weeks at a dose of $3 \mathrm{mg} / \mathrm{kg}$. The treatments were continued until disease progression, intolerable toxicity, patient refusal of further treatment, or death from any cause.

\section{Evaluation}

Baseline demographics and clinical characteristics were summarized by treatment group. It has been reported that the prognosis of patients with HCC depends on both residual liver function and the extent of the tumor. ${ }^{16-18}$ Child-Pugh classification and standard liver function tests were used to evaluate remaining liver function. Clinically significant portal hypertension, defined by a hepatic venous pressure gradient $\geq 10 \mathrm{mmHg}$, was estimated by evidence of overt clinical decompensation (i.e., ascites, varices, or hepatic encephalopathy) or presence of splenomegaly with thrombocytopenia (platelet count $<150,000 / \mathrm{mm}^{3}$ ). ${ }^{19}$ Pretreatment intrahepatic

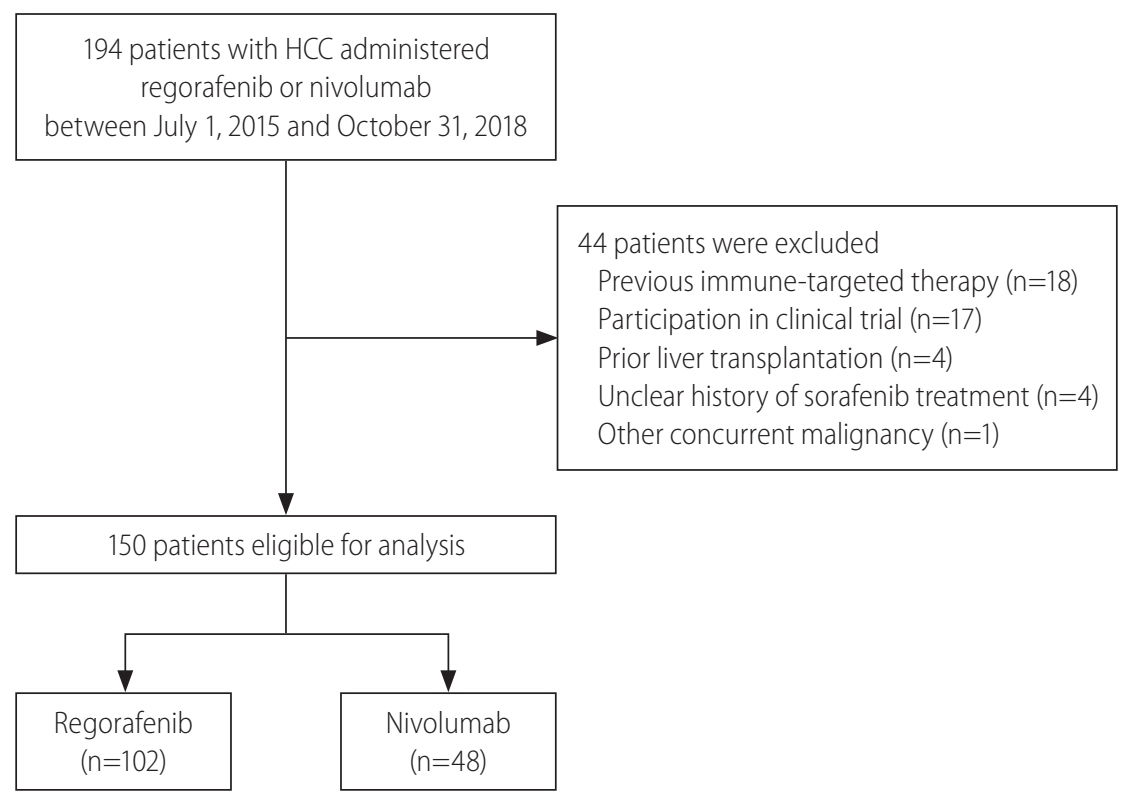

Figure 1. Flow diagram summarizing the disposition process of patients. HCC, hepatocellular carcinoma. 
Table 1. Baseline characteristics by treatment group

\begin{tabular}{|c|c|c|c|c|}
\hline \multirow{2}{*}{ Characteristic } & \multirow{2}{*}{ Overall $(n=150)$} & \multicolumn{2}{|c|}{ Group } & \multirow{2}{*}{$P$-value } \\
\hline & & Regorafenib $(n=102)$ & Nivolumab $(n=48)$ & \\
\hline Age (years) & $62(55-70)$ & $62(56-71)$ & $61(54-67)$ & 0.14 \\
\hline Male & $122(81.3)$ & $83(81.4)$ & $39(81.2)$ & 0.99 \\
\hline HCC etiology & & & & 0.44 \\
\hline HBV & $124(82.7)$ & $86(84.3)$ & $38(79.2)$ & \\
\hline Others & $26(17.3)$ & $16(15.7)$ & $10(20.8)$ & \\
\hline Child-Pugh score & & & & 0.003 \\
\hline 5 & $86(57.3)$ & $66(64.7)$ & $20(41.7)$ & \\
\hline 6 & $51(34.0)$ & $32(31.4)$ & 19 (39.6) & \\
\hline $7-9$ & $13(8.7)$ & $4(3.9)$ & $9(18.8)$ & \\
\hline Vascular invasion & $66(44.0)$ & $42(41.2)$ & $24(50.0)$ & 0.31 \\
\hline Biliary invasion & $7(4.7)$ & $5(4.9)$ & $2(4.2)$ & 0.84 \\
\hline Intrahepatic tumor burden & & & & 0.40 \\
\hline None & $25(16.7)$ & 19 (18.6) & $6(12.5)$ & \\
\hline$<50 \%$ & $93(62.0)$ & $64(62.7)$ & $29(60.4)$ & \\
\hline$\geq 50 \%$ & $32(21.3)$ & $19(18.6)$ & $13(27.1)$ & \\
\hline Extrahepatic metastases & $120(80.0)$ & $79(77.5)$ & $41(85.4)$ & 0.26 \\
\hline Lymph node & $54(36.0)$ & $37(36.3)$ & $17(35.4)$ & \\
\hline Lung & $77(51.3)$ & $46(45.1)$ & $31(64.6)$ & \\
\hline Bone & $32(21.3)$ & $21(20.6)$ & $11(22.9)$ & \\
\hline Peritoneum & $24(16.0)$ & $16(15.7)$ & $8(16.7)$ & \\
\hline Others & $3(2.0)$ & $1(1.0)$ & $2(4.2)$ & \\
\hline Portal hypertension & $64(42.7)$ & $42(41.2)$ & $22(45.8)$ & 0.59 \\
\hline BCLC stage & & & & 0.56 \\
\hline B & $5(3.3)$ & $4(3.9)$ & $1(2.1)$ & \\
\hline C & $145(96.7)$ & $98(96.1)$ & 47 (97.9) & \\
\hline \multicolumn{5}{|l|}{ Laboratory data } \\
\hline Albumin (g/dL) & $3.7(3.3-4.0)$ & $3.8(3.4-4.1)$ & $3.7(3.2-3.9)$ & 0.08 \\
\hline Total bilirubin (mg/dL) & $1.0(0.7-1.4)$ & $1.0(0.7-1.3)$ & $1.1(0.7-1.7)$ & 0.29 \\
\hline AST (IU/L) & $49(33-80)$ & $45(32-77)$ & $60(39-90)$ & 0.16 \\
\hline ALT (IU/L) & $37(22-59)$ & $37(22-57)$ & $38(23-60)$ & 0.42 \\
\hline ALP (IU/L) & $138(88-220)$ & $133(87-220)$ & $144(92-212)$ & 0.88 \\
\hline Creatinine (mg/dL) & $0.81(0.68-0.93)$ & $0.82(0.68-0.93)$ & $0.80(0.69-0.93)$ & 0.44 \\
\hline Platelet count $\left(\times 10^{9} / \mathrm{L}\right)$ & $154(105-218)$ & $164(107-228)$ & $149(101-204)$ & 0.28 \\
\hline INR & $1.08(1.02-1.15)$ & $1.06(1.00-1.12)$ & $1.10(1.04-1.21)$ & 0.002 \\
\hline $\operatorname{AFP}(\mathrm{ng} / \mathrm{mL})$ & $431(12.5-4,185.0)$ & $338.0(11.9-3,799.3)$ & $760.0(18.4-4,665.0)$ & 0.90 \\
\hline PIVKA (mAU/mL) & $1,453(135-8,898)$ & $1,365(149-8,699)$ & $1,803(107-10,545)$ & 0.48 \\
\hline MoRAL & $570.6(205.1-1,254.3)$ & $570.6(240.1-1,224.0)$ & $559.5(234.2-1,276.5)$ & 0.62 \\
\hline Intolerant to sorafenib & $13(8.7)$ & $6(5.9)$ & $7(14.6)$ & 0.08 \\
\hline Duration of sorafenib* (months) & $2.8(2.0-4.7)$ & $3.0(2.3-6.2)$ & $2.5(1.4-3.1)$ & $<0.001$ \\
\hline $\begin{array}{l}\text { Time interval between sorafenib } \\
\text { and treatment (months) }\end{array}$ & $1.2(0.0-4.7)$ & $0.9(0.0-4.6)$ & $1.8(0.3-5.8)$ & 0.89 \\
\hline
\end{tabular}

Values are presented as median (interquartile range) or number (\%) of patients.

HCC, hepatocellular carcinoma; HBV, hepatitis B virus; BCLC, Barcelona Clinic Liver Cancer; AST, aspartate aminotransferase; ALT, alanine aminotransferase; ALP, alkaline phosphatase; INR, international normalized ratio; AFP, alpha-fetoprotein; PIVKA, protein induced by vitamin K absence or antagonist; MoRAL, model to predict tumor recurrence after living donor liver transplantation.

${ }^{*}$ Assessed for patients who progressed after sorafenib $(n=136)$. 


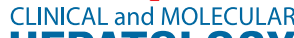

\section{HEPATOLOGY}

tumor burden and extrahepatic spread were assessed based on an imaging study prior to treatment initiation. A model to predict tumor recurrence after living donor liver transplantation score $(=11 \times \sqrt{\text { protein induced by vitamin } \mathrm{K} \text { absence- } I}+2 \times \sqrt{\text { alpha- }}$ fetoprotein [AFP]), which was developed and validated previously to have prognostic value in patients undergoing liver transplantation for HCC was calculated for the individual patient and included in the further analyses..$^{20,21}$

\section{Clinical outcome measures}

The date of the first regorafenib or nivolumab administration was assigned as the index date. The primary endpoint was OS, defined as time from the index date to death from any cause. For patients who switched to the other treatment (i.e., regorafenib after nivolumab or nivolumab after regorafenib), the follow-up was censored at the date of initiation of the subsequent treatment. Secondary endpoints were time to progression (TTP) defined as time from the index date to radiologic progression, objective response rate, duration of response, disease control rate. To evaluate radiologic response, two independent radiologists reviewed the images (abdomen computed tomography or magnetic resonance imaging), and the clinicians read the same images independently based on modified RECIST (mRECIST). ${ }^{22}$ Every patient underwent scheduled imaging follow-up every 2-3 months. Considering insufficient drug exposure, imaging studies within 4 weeks of treatment initiation were precluded for the assessment of tumor response. Subgroup analyses were conducted for the

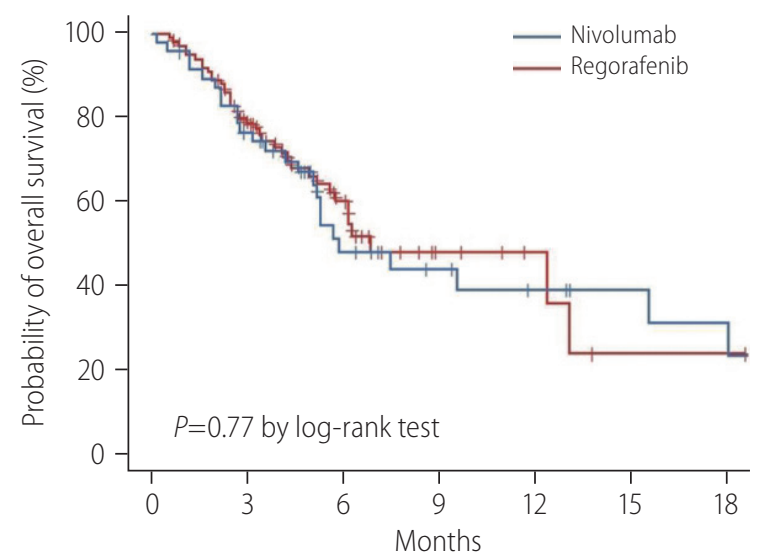

No. at risk

$\begin{array}{llllllll}\text { Nivolumab } & 48 & 35 & 15 & 10 & 7 & 5 & 4\end{array}$
$\begin{array}{lllllll}\text { Regorafenib } & 102 & 70 & 25 & 7 & 4 & 1\end{array}$ primary and secondary endpoints according to baseline characteristics. Treatment-related toxicities leading to premature discontinuation of the treatment before the documentation of disease progression were investigated.

\section{Statistical analyses}

Baseline demographic and clinical characteristics were compared between treatment groups. For group-wise comparisons, the independent samples $t$-test was used for continuous variables and either the chi-square test or the Fischer's exact test was applied for categorical variables.

The Kaplan-Meier method was used for analyses of OS and TTP. Differences between treatment groups were verified by log-rank test. To investigate whether results were confounded by other risk factors for OS or TTP, we used the multivariable Cox regression analysis. Variables in the multivariable analyses were selected using stepwise regression with the forward selection method. The objective response rate and disease control rate in each group were compared using the Cochran-Mantel-Haenszel test. Inverse probability treatment weighting (IPTW) based on the propensity score was applied to mitigate baseline differences between the groups. ${ }^{23,24}$ The propensity score for each patient was calculated using a logistic regression model using baseline characteristics, such as age, Child-Pugh score, and tumor stage. Moreover, we employed weighted Cox proportional hazards regression model to identify the treatment effect on OS and TTP.

All statistical analyses were performed using $R$ software (version

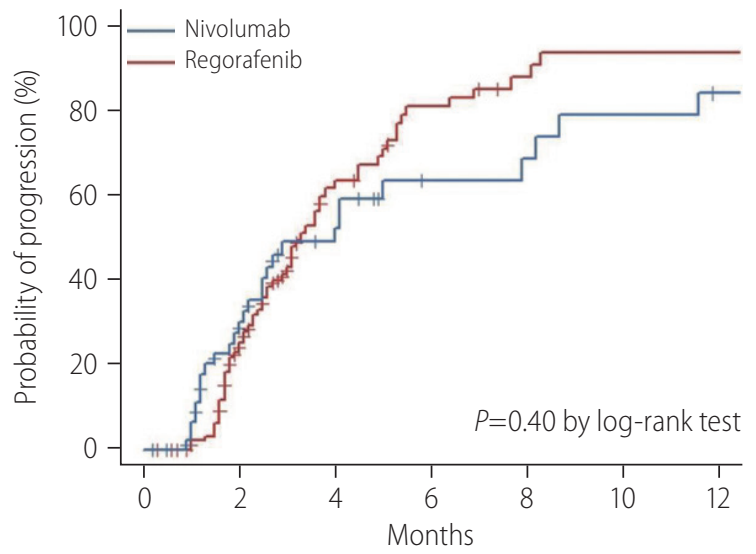

No. at risk

Nivolumab

Regorafenib

$\begin{array}{lllllll}48 & 29 & 15 & 7 & 6 & 4 & 2 \\ 102 & 63 & 21 & 9 & 4 & 2 & 2\end{array}$

B

Figure 2. Kaplan-Meier curves of OS (A) and TTP (B). OS, overall survival; TTP, time to progression. 
3.6.0; R Foundation for Statistical Computing, Vienna, Austria), and IBM SPSS Statistics version 25 (IBM Corporation, Armonk, NY, USA). A two-tailed $P$ value less than 0.05 was considered statistically significant.

\section{RESULTS}

\section{Patient population}

Among 150 HCC patients included in final analyses, 102 pa- tients were treated with regorafenib and 48 received nivolumab. The baseline demographic and clinical characteristics of the study patients by the group are shown in Table 1. The median age was 62 (interquartile range [IQR], 55-70) years overall, and was not significantly different between the groups $(P=0.14)$. Male patients accounted for $81.3 \%$ of patients, and most study patients (82.7\%) were chronically infected with hepatitis B virus. Almost all patients (96.7\%) were Barcelona Clinic Liver Cancer stage C, and there was no statistical difference between the groups. While 25 patients (16.7\%) had no intrahepatic tumor, $>50 \%$ of the liver was occupied with the tumor in 32 patients $(21.3 \%)$ at baseline.

Table 2. Univariable and multivariable Cox regression analyses for overall survival

\begin{tabular}{|c|c|c|c|c|}
\hline \multirow{2}{*}{ Characteristic } & \multicolumn{2}{|c|}{ Univariable analysis } & \multicolumn{2}{|c|}{ Multivariable analysis } \\
\hline & $\mathrm{HR}(95 \% \mathrm{Cl})$ & $P$-value & $\mathrm{HR}(95 \% \mathrm{Cl})$ & $P$-value \\
\hline Nivolumab (vs. regorafenib) & $1.081(0.644-1.813)$ & 0.77 & $0.536(0.300-0.957)$ & 0.04 \\
\hline Age (per year increase) & $0.987(0.966-1.009)$ & 0.24 & & \\
\hline Male sex (vs. female) & $2.313(1.052-5.086)$ & 0.04 & $2.587(1.140-5.872)$ & 0.02 \\
\hline Etiology of HCC, HBV (vs. others) & $1.572(0.775-3.185)$ & 0.21 & & \\
\hline Child-Pugh score & & $<0.001$ & & $<0.001$ \\
\hline 5 & 1 (reference) & & 1 (reference) & \\
\hline 6 & $3.532(2.009-6.210)$ & $<0.001$ & $3.458(1.912-6.252)$ & $<0.001$ \\
\hline $7-9$ & $6.264(2.926-13.410)$ & $<0.001$ & $5.195(2.073-13.018)$ & $<0.001$ \\
\hline Vascular invasion, yes (vs. no) & $2.104(1.275-3.471)$ & 0.004 & & \\
\hline Biliary invasion, yes (vs. no) & $3.049(1.205-7.718)$ & 0.02 & & \\
\hline Intrahepatic tumor burden & & $<0.001$ & & 0.01 \\
\hline None & 1 (reference) & & 1 (reference) & \\
\hline$<50 \%$ & $2.024(0.847-4.833)$ & 0.11 & $1.488(0.595-3.722)$ & 0.40 \\
\hline$\geq 50 \%$ & $7.148(2.853-17.905)$ & $<0.001$ & $2.801(1.019-7.703)$ & 0.046 \\
\hline Extrahepatic metastasis, yes (vs. no) & $0.522(0.296-0.919)$ & 0.02 & & \\
\hline Portal hypertension, yes (vs. no) & $1.503(0.919-2.458)$ & 0.10 & & \\
\hline BCLC stage C (vs. B) & $0.913(0.222-3.759)$ & 0.90 & & \\
\hline AST (per IU/L) & $1.012(1.008-1.016)$ & $<0.001$ & $1.007(1.002-1.012)$ & 0.004 \\
\hline ALT (per IU/L) & $1.008(1.004-1.012)$ & $<0.001$ & & \\
\hline ALP (per IU/L) & $1.003(1.002-1.004)$ & $<0.001$ & & \\
\hline Creatinine (per mg/dL) & $0.359(0.113-1.142)$ & 0.08 & & \\
\hline Platelet count (per $\times 10^{9} / \mathrm{L}$ ) & $1.002(1.000-1.005)$ & 0.09 & & \\
\hline AFP $\geq 400 \mathrm{ng} / \mathrm{mL}$ & $1.359(0.828-2.232)$ & 0.23 & & \\
\hline PIVKA $\geq 1,000 \mathrm{mAU} / \mathrm{mL}$ & $2.842(1.622-4.980)$ & $<0.001$ & & \\
\hline MoRAL $\geq 314.8$ & $3.056(1.677-5.569)$ & $<0.001$ & $1.897(0.999-3.602)$ & 0.05 \\
\hline
\end{tabular}

$P$ values were determined using Cox proportional hazards regression models. $P<0.05$ indicated a significant difference.

HR, hazards ratio; $\mathrm{Cl}$, confidence interval; HCC, hepatocellular carcinoma; HBV, hepatitis B virus; BCLC, Barcelona Clinic Liver Cancer; AST, aspartate aminotransferase; ALT, alanine aminotransferase; ALP, alkaline phosphatase; AFP, alpha-fetoprotein; PIVKA, protein induced by vitamin K absence or antagonist; MoRAL, model to predict tumor recurrence after living donor liver transplantation. 
Most patients (91.3\%) were Child-Pugh class A, and the proportion of patients with Child-Pugh class B was significantly higher in the nivolumab group $(P=0.003)$. Prothrombin time in international normalized ratio was significantly higher in the nivolumab group $(P=0.002)$. Mean serum albumin levels were lower in the nivolumab group, but the difference was not statistically significant $(P=0.08)$. Thirteen $(8.7 \%)$ patients who were intolerant to sorafenib treatment were included in this study. The proportion of sorafenib-intolerant patients was higher in the nivolumab group than in the regorafenib group ( $14.6 \%$ vs. $5.9 \%$ ).

During the follow-up period, all of the patients in the nivolumab group received prespecified fixed dose. There was only one patient $(2 \%)$ who experienced treatment interruption. Sixty-five patients $(64 \%)$ required dose modification or treatment interruption due to adverse events in the regorafenib group. Excluding treatment delays or interruptions, 60 patients (59\%) received full dose without dose reduction, and 22 patients (22\%) received reduced dose $(<120 \mathrm{mg})$ of regorafenib.

\section{OS}

At the cutoff date for the final analysis (January 15, 2019), the median duration of follow-up of the entire cohort was 5.0 months (IQR, 2.9-7.5): 4.3 months (IQR, 2.9-6.6) for the regorafenib group and 5.2 months (IQR, 3.1-9.7) for the nivolumab group. Overall, 65 patients (43.3\%) died during the follow-up period: 38

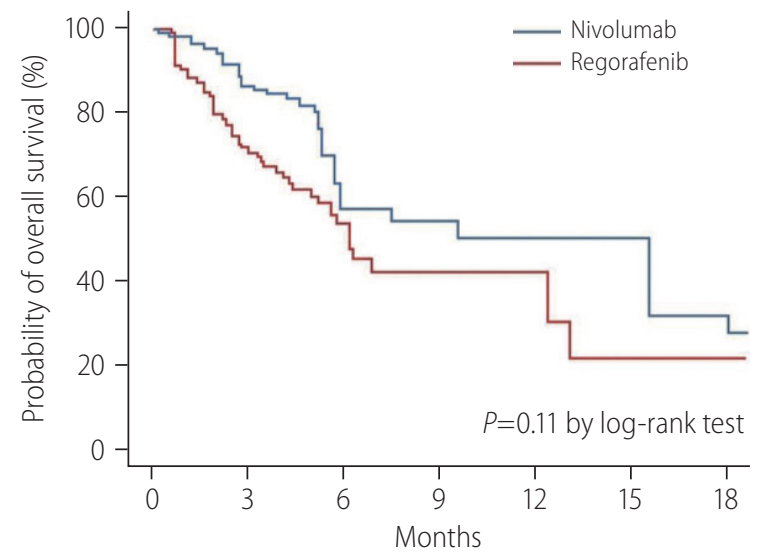

No. at risk

$\begin{array}{llllllll}\text { Nivolumab } & 48 & 35 & 15 & 10 & 7 & 5 & 4\end{array}$ $\begin{array}{llllllll}\text { Regorafenib } & 102 & 70 & 25 & 7 & 4 & 1 & 1\end{array}$
(37.3\%) of 102 patients in the regorafenib group and 27 (56.3\%) of 48 patients in the nivolumab group. Median OS was 5.9 months (95\% confidence interval [CI], 3.2-18.1) in the nivolumab group and 6.9 months $(95 \% \mathrm{Cl}, 3.5-13.1)$ months in the regorafenib group ( $P=0.77$ by log-rank test) (Fig. $2 A)$. In the multivariable analysis, treatment with nivolumab was independently associated with improved OS compared to regorafenib after adjusting for confounding factors, including Child-Pugh score and intrahepatic tumor burden (adjusted hazard ratio [aHR], 0.54; $95 \% \mathrm{Cl}$, 0.30-0.96; $P=0.04$ ) (Table 2). After balancing the baseline characteristics by IPTW, there was no longer any significant difference between the treatment groups (Supplementary Table 1). Although there was no statistically significant difference between the groups, nivolumab was associated with better OS than regorafenib ( $P=0.11$ by log-rank test) (Fig. $3 A$ ). Weighted Cox proportional hazards model also revealed that the nivolumab treatment was associated with improved OS (vs. regorafenib; aHR, 0.34; 95\% Cl, 0.18-0.65; $P=0.001$ ) (Supplementary Table 2). Similar results were observed in additional analysis without censoring the patient who switched to the other treatment (Supplementary Fig. 1).

\section{TTP}

During the follow-up period, tumors progressed radiologically in 91 (60.7\%) of 150 patients based on mRECIST: 62 (60.8\%) of 102 patients in the regorafenib group and $29(60.4 \%)$ of 48 patients

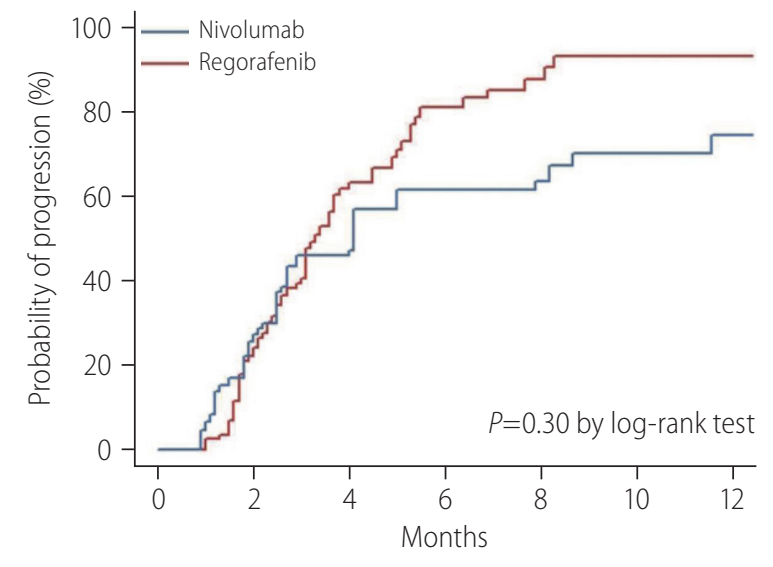

No. at risk

$\begin{array}{llllllll}\text { Nivolumab } & 48 & 29 & 15 & 7 & 6 & 4 & 2\end{array}$ $\begin{array}{llllllll}\text { Regorafenib } & 102 & 63 & 21 & 9 & 4 & 2 & 2\end{array}$

B

Figure 3. Kaplan-Meier curves of OS (A) and TTP (B) after IPTW. OS, overall survival; TTP, time to progression; IPTW, inverse probability treatment weighting. 
in the nivolumab group. The median TTP was not significantly different between the nivolumab group (4.0 months; $95 \% \mathrm{Cl}, 1.8-$ 8.7) and the regorafenib group (3.3 months; $95 \% \mathrm{Cl}, 2.0-5.3)$ ( $P=0.40$ by log-rank test) (Fig. $2 \mathrm{~B})$. In the multivariable analysis, there was no significant association with improved TTP between treatments (nivolumab vs. regorafenib; aHR, $0.81 ; 95 \% \mathrm{Cl}, 0.51-$ 1.30; $P=0.48$ ) (Table 3). After IPTW, there was no significant difference in TTP between the groups ( $P=0.30$ by log-rank test) (Fig. $3 \mathrm{~B})$, and treatment group was not an independent predictor for TTP (nivolumab vs. regorafenib; aHR, 0.74; 95\% Cl, 0.39-1.41; $P=0.36$ ) (Supplementary Table 3).

\section{Overall tumor response}

No patient in either treatment group achieved a complete response. Eight (16.7\%) of 48 patients in the nivolumab group and six $(5.9 \%)$ of 102 patients in the regorafenib group achieved a partial response by $m R E C I S T$, and nivolumab showed significantly better objective response rate compared to regorafenib $(P=0.041)$ (Table 4). The disease control rate (i.e., the proportion of patients who had an objective response or disease stabilization) was $50.0 \%$ in the nivolumab group and $47.1 \%$ in the regorafenib group $(P=0.58)$. Among the 14 patients who achieved an objec-

Table 3. Univariable and multivariable Cox regression analyses for time to progression

\begin{tabular}{|c|c|c|c|c|}
\hline \multirow{2}{*}{ Characteristic } & \multicolumn{2}{|c|}{ Univariable analysis } & \multicolumn{2}{|c|}{ Multivariable analysis } \\
\hline & HR $(95 \% \mathrm{Cl})$ & $P$-value & $\mathrm{HR}(95 \% \mathrm{Cl})$ & $P$-value \\
\hline Nivolumab (vs. regorafenib) & $0.824(0.522-1.299)$ & 0.40 & $0.812(0.509-1.297)$ & 0.48 \\
\hline Age (per year increase) & $0.985(0.964-1.006)$ & 0.15 & & \\
\hline Male sex (vs. female) & $1.375(0.809-2.335)$ & 0.24 & & \\
\hline Etiology of HCC, HBV (vs. others) & $1.458(0.792-2.681)$ & 0.23 & & \\
\hline Child-Pugh score & & 0.20 & & \\
\hline 5 & 1 (reference) & & & \\
\hline 6 & $1.502(0.954-2.366)$ & 0.08 & & \\
\hline $7-9$ & $0.972(0.351-2.689)$ & 0.96 & & \\
\hline Vascular invasion, yes (vs. no) & $1.016(0.663-1.558)$ & 0.94 & & \\
\hline Biliary invasion, yes (vs. no) & $1.666(0.672-4.131)$ & 0.27 & & \\
\hline Intrahepatic tumor burden & & 0.04 & & \\
\hline None & 1 (reference) & & & \\
\hline$<50 \%$ & $1.098(0.642-1.878)$ & 0.73 & & \\
\hline$\geq 50 \%$ & $2.067(1.073-3.982)$ & 0.03 & & \\
\hline Extrahepatic metastasis, yes (vs. no) & $0.810(0.475-1.379)$ & 0.44 & & \\
\hline Portal hypertension, yes (vs. no) & $1.332(0.869-2.041)$ & 0.19 & & \\
\hline BCLC stage C (vs. B) & $0.305(0.109-0.856)$ & 0.02 & & \\
\hline AST (per IU/L) & $1.006(1.001-1.011)$ & 0.01 & & \\
\hline ALT (per IU/L) & $1.003(0.998-1.009)$ & 0.23 & & \\
\hline ALP (per IU/L) & $1.002(1.001-1.003)$ & 0.001 & $1.002(1.001-1.003)$ & 0.002 \\
\hline Creatinine (per mg/dL) & $0.589(0.230-1.510)$ & 0.27 & & \\
\hline Platelet count (per $\times 10^{9} / \mathrm{L}$ ) & $1.003(1.001-1.006)$ & 0.004 & $1.003(1.001-1.005)$ & 0.01 \\
\hline AFP $\geq 400 \mathrm{ng} / \mathrm{mL}$ & $1.063(0.703-1.608)$ & 0.77 & & \\
\hline PIVKA $\geq 1,000 \mathrm{mAU} / \mathrm{mL}$ & $1.217(0.803-1.846)$ & 0.36 & & \\
\hline MoRAL $\geq 314.8$ & $1.210(0.794-1.844)$ & 0.37 & & \\
\hline
\end{tabular}

$P$ values were determined using Cox proportional hazards regression models. $P<0.05$ indicated a significant difference.

HR, hazards ratio; $\mathrm{Cl}$, confidence interval; HCC, hepatocellular carcinoma; HBV, hepatitis B virus; BCLC, Barcelona Clinic Liver Cancer; AST, aspartate aminotransferase; ALT, alanine aminotransferase; ALP, alkaline phosphatase; AFP, alpha-fetoprotein; PIVKA, protein induced by vitamin $\mathrm{K}$ absence or antagonist; MoRAL, model to predict tumor recurrence after living donor liver transplantation. 
Table 4. Best overall response by treatment group

\begin{tabular}{lcc}
\hline & $\begin{array}{c}\text { Regorafenib } \\
(\mathbf{n = 1 0 2 )}\end{array}$ & $\begin{array}{c}\text { Nivolumab } \\
(\mathbf{n}=\mathbf{4 8})\end{array}$ \\
\hline $\begin{array}{l}\text { Best overall response* } \\
\text { Complete response }\end{array}$ & 0 & 0 \\
Partial response & $6(5.9)$ & $8(16.7)$ \\
Stable disease & $42(41.2)$ & $16(33.3)$ \\
Progressive disease & $37(36.3)$ & $17(35.4)$ \\
Not assessed & $17(16.7)$ & $7(14.6)$ \\
Objective response $^{\dagger}$ & $6(5.9)$ & $8(16.7)$ \\
Disease control $^{\ddagger}$ & $48(47.1)$ & $24(50.0)$ \\
\hline
\end{tabular}

Values are presented as number (\%) of patients.

*Based on radiological review using modified Response Evaluation Criteria in Solid Tumors (mRECIST) for hepatocellular carcinoma.

${ }^{\dagger}$ Two-sided $P=0.041$.

${ }^{\ddagger}$ Two-sided $P=0.58$.

tive response, 10 patients did not progress during the follow-up. Median duration of response, which was evaluated for patients with tumor response, was 4.2 months (3.5-not estimable [NE]) in the nivolumab group and 2.7 (2.2-NE) months in the regorafenib group ( $P=0.73$ by log-rank test).

\section{Subgroup analysis}

Comparisons of outcomes between treatments were assessed among the subgroups. There was no difference in OS or TTP between the groups regarding duration of prior sorafenib treatment (Supplementary Fig. 2), or Child Pugh score (Supplementary Fig. 3). Further analysis according to the presence or absence of portal hypentension/macrovascular invasion, and AFP level showed no significant OS difference between the two treatment groups (Supplementary Fig. 4).

\section{Safety}

The reasons for the drug discontinuation were assessed for the safety analysis. Eighty-three patients discontinued the treatment due to disease progression among 122 patients who discontinued the treatment (Supplementary Table 4). Adverse events that caused premature drug discontinuation occurred in 24 patients $(23.5 \%)$ in the regorafenib group and eight patients $(16.7 \%)$ in the nivolumab group $(P=0.34)$. The major cause of drug discontinuation before radiologic progression was hepatic decompensation: four $(8.3 \%)$ in the nivolumab group and $10(9.8 \%)$ in the regorafenib group $(P=0.77)$. Subsequent treatments after study drug failure were assessed. The proportion of patients who received any treatments after the study drug failure in both groups was similar ( $P=0.54)$ (Supplementary Table 5).

\section{DISCUSSION}

In the current study in HCC patients who had experienced sorafenib treatment failure, nivolumab treatment was associated with improved OS compared with regorafenib treatment. The risk of all-cause mortality was reduced by $46 \%$ in patients receiving nivolumab compared to patients receiving regorafenib. Although TTP did not differ significantly between the treatments, nivolumab demonstrated a significantly better objective response rate than regorafenib. Both drugs were well tolerated during the treatment period; however, adverse events leading to drug discontinuation before radiologic disease progression were more frequent in patients receiving regorafenib.

To date, this is the first study in a real-world setting comparing the effectiveness of regorafenib and nivolumab treatments in HCC patients who previously failed sorafenib treatment. Nivolumab was more effective than regorafenib in terms of OS. While median OS was longer in patients treated with regorafenib (6.9 months) versus nivolumab (5.9 months), nivolumab showed a significant association with improved OS after adjusting for confounders. In the present study, patients with higher Child-Pugh scores at baseline, an independent predictive factor for poor OS, were more likely to be treated with nivolumab. To minimize treatment selection bias, we adopted IPTW in addition to multivariable analyses, and the association of nivolumab with prolonged OS was confirmed.

The higher objective response rate with nivolumab might confer its association with improved OS compared with regorafenib. The objective response rate with nivolumab was $16.7 \%$ in the current study, a finding that is consistent with previous studies reporting objective response rates of $14.3-16.7 \% .^{10,14,25}$ However, the objective response rate with regorafenib $(5.9 \%)$ was lower compared to the previous studies (10.0-10.6\%)., ${ }^{5,15}$ In the RESORCE trial, the objective response rate was $10.6 \%$, and $68 \%$ of patients experienced drug interruption or dose modification during the treatment. ${ }^{5}$ The rates of treatment interruption or dose modification of patients receiving regorafenib in our present study was similar to those in the RESORCE trial. Dose intensity was also comparable. Scheduled imaging was performed every 6 weeks at 
Cheol-Hyung Lee, et al. Nivolumab versus regorafenib for advanced HCC

the beginning of treatment in the RESORCE trial. However the imaging intervals were variable ranged from 2 to 3 months in the current retrospective study, which may have affected the tumor response._The duration of response was longer in patients treated with nivolumab than in patients treated with regorafenib, even though the difference was not statistically significant. This finding is consistent with the results of the CheckMate 040 trial which reported sustained tumor response with nivolumab in HCC patients. ${ }^{6,10}$ Further studies with sufficient observational periods are warranted to demonstrate a statistically significant difference in the duration of response between nivolumab and regorafenib.

The median OS with nivolumab (5.9 months) and regorafenib (6.9 months) was shorter than reported in the corresponding results from the pivotal trials. Median OS with regorafenib was 10.7 months in the RESORCE trial, and median OS with nivolumab was 15.1 months in the sorafenib progressor cohort in the CheckMate 040 trial. $^{9,10}$ In contrast, recent real-world evidence studies evaluating the efficacy of nivolumab reported that median OS with nivolumab ranged from $<2$ months to 11.0 months. ${ }^{11-14}$ Considering the major causes of death of advanced HCC patients were intrahepatic tumor progression and complications of cirrhosis, the discrepancy between the results of clinical trials and real-world studies can be explained by the differences in baseline characteristics of the study population. ${ }^{16,18}$ However, even though regorafenib treatment demonstrated efficacy over placebo in prolonging OS after sorafenib failure in the RESORCE trial, and the ability of nivolumab to improve OS compared to regorafenib was demonstrated in the current study, survival gain with nivolumab treatment is still unsatisfactory. Recently, the efficacy of combination treatments with immune checkpoint inhibitors plus molecular targeted agents in advanced HCC patients was evaluated in phase $1 \mathrm{~b}$ trials. ${ }^{26,27}$ Promising results of objective response rates over $30-40 \%$ were reported; however, safety-related issues need to be clarified. In a recent study, it was reported that less than half of patients were eligible for subsequent systemic therapy after sorafenib failure due to impaired hepatic function. ${ }^{28}$ Therefore, it is crucial to establish a treatment strategy maximizing efficacy without compromising safety.

Despite the unsatisfactory effects, it is noteworthy that small portion of patients did achieve prolonged DOR more than a year and even complete responses with regorafenib or nivolumab treatment. HCC patients who experienced sorafenib failure are heterogenous in terms of varying prior treatments, ${ }^{29}$ different tumor characteristics, and degrees of liver fibrosis, but biomarker based treatment is limited due to the risk of liver biopsy. Thus, treatment strategy of those patients is mainly dependent on clinical judgment. Previous study by Lee et al. ${ }^{30}$ estimated survival and prognosis of patients who experienced sorafenib failure based on clinical parameters. In our study, male sex, extensive intraheptatic tumor burden and higher Child-Pugh score at the time of sorafenib failure were identified as poor survival markers.

The safety profiles of regorafenib and nivolumab in the present study were generally consistent with those reported in previous studies. ${ }^{5,6,13-15,25}$ Whilst both treatments showed comparable safety in terms of drug discontinuation rate, the proportion of patients with impaired hepatic function was significantly higher among patients receiving nivolumab compared to regorafenib at baseline. Furthermore, three of four patients who discontinued nivolumab treatment due to hepatic decompensation had extensive intrahepatic tumor burden (>50\%) at the start of treatment. Therefore, the possibility of overestimating the hepatotoxicity of nivolumab should be accounted for. Previous studies including Child-Pugh class B patients with HCC showed similar safety profile of nivolumab between Child-Pugh class $A$ and Child-Pugh Class B subgroups. ${ }^{14,25}$ Moreover, one patient with Child-Pugh class $C$ disease at baseline showed complete response during the treatment with nivolumab in a small study involving 14 HCC patients. ${ }^{31}$ The safety of nivolumab in patients with hepatic impairment needs to be validated in future studies.

Our study has several limitations to be considered. First, in terms of tumor response assessment, the follow-up intervals for imaging studies varied among the study patients. However, since this issue results from the nature of the retrospective study design and was not limited to either regorafenib or nivolumab treatment, the effect on the efficacy comparison is thought to be negligible. Second, although the major adverse events leading to treatment discontinuation were analyzed, it was impossible to obtain accurate information about adverse events that occurred during the study period. Further, well-designed prospective studies are needed to verify the safety in real-world clinical practice. Third, because we assessed the tumor response based on mRECIST, pseudoprogression, a unique pattern of tumor response with immunotherapy, was not considered for the patients receiving nivolumab. Though the rate of pseudoprogression is known to be rare $(<10 \%)$ across tumor types, ${ }^{32}$ there have been reports of prolonged response to immune checkpoint inhibitors after pseudoprogression in HCC patients. ${ }^{33,34}$ In the era of cancer immunotherapy, tumor response evaluation based on immune RECIST should be further validated in HCC patients. ${ }^{35}$

In the current study in patients with HCC who previously failed 
sorafenib treatment, subsequent nivolumab treatment may improve OS over regorafenib treatment and also demonstrated a higher objective response rate. Since nivolumab, an immune modulator, has a completely different mechanism of action and side effect profile from sorafenib, treatment with nivolumab may be a potential therapeutic option for the treatment of HCC after sorafenib failure, particularly in patients showing sorafenib intolerance. Both regorafenib and nivolumab were well tolerated overall. Additional studies investigating the effectiveness of nivolumab in combination with other treatments are warranted in consideration of its efficacy and safety.

\section{Author's contribution}

The corresponding author (Yoon Jun Kim) had full access to all the data in the study and takes responsibility for the integrity of the data and the accuracy of the data analysis.

1) Study concept and design: Cheol-Hyung Lee, Yun Bin Lee, and Yoon Jun Kim

2) Provision of study materials or patients: Yun Bin Lee, Eun Ju Cho, Kyung-Hun Lee, Jeong-Hoon Lee, Su Jong Yu, Tae-You Kim, Jung-Hwan Yoon, and Yoon Jun Kim

3) Collection and assembly of data: Cheol-Hyung Lee, Minseok Albert Kim, Heejoon Jang, Hyunwoo Oh, Sun Woong Kim

4) Data analysis and interpretation: Cheol-Hyung Lee, Yun Bin Lee, and Yoon Jun Kim

5) Manuscript writing: Cheol-Hyung Lee, Yun Bin Lee, and Yoon Jun Kim

6) Final approval of manuscript: All authors

\section{Acknowledgements}

This research was supported by the Seoul National University Hospital Research Fund (grant No. 03-2019-0100).

\section{Conflicts of Interest}

Dr. Lee YB reports receiving research grant from Samjin Pharmaceuticals; Dr. Lee KH reports honoraria from Roche and AstraZeneca, and serves an advisory role for AstraZeneca, Samsung Bioepis, BAYER, Roche, Eisai, and Ono Pharmaceutical; Dr. Lee JH reports receiving lecture fee from GreenCross Cell, Daewoong Pharmaceuticals, and Gilead Korea; Dr. Yu SJ reports lecture fee from Bayer HealthCare Pharmaceuticals; Dr. Kim YJ, research grants from Bristol-Myers Squibb, Roche, JW Creagene, Bukwang Pharmaceuticals, Handok Pharmaceuticals, Hanmi Pharmaceuticals, Yuhan Pharmaceuticals, Samjin Pharmaceuticals, AstraZeneca, and Pharmaking, and lecture fees from Bayer HealthCare
Pharmaceuticals, Gilead Science, MSD Korea, Yuhan Pharmaceuticals, Samil Pharmaceuticals, CJ Pharmaceuticals, Bukwang Pharmaceuticals, and Handok Pharmaceuticals; Dr. Yoon JH, research grants from AstraZeneca, Bayer HealthCare Pharmaceuticals, Daewoong Pharmaceuticals, and Bukwang Pharmaceuticals. No other potential conflict of interest relevant to this article was reported.

\section{SUPPLEMENTARY MATERIAL}

Supplementary material is available at Clinical and Molecular Hepatology website (http://www.e-cmh.org).

\section{REFERENCES}

1. Bray F, Ferlay J, Soerjomataram I, Siegel RL, Torre LA, Jemal A. Global cancer statistics 2018: GLOBOCAN estimates of incidence and mortality worldwide for 36 cancers in 185 countries. CA Cancer J Clin 2018;68:394-424.

2. Liu Z, Jiang Y, Yuan H, Fang Q, Cai N, Suo C, et al. The trends in incidence of primary liver cancer caused by specific etiologies: results from the global burden of disease study 2016 and implications for liver cancer prevention. J Hepatol 2019;70:674-683.

3. Villanueva A. Hepatocellular carcinoma. N Engl J Med 2019;380: 1450-1462.

4. Dika IE, Abou-Alfa GK. Treatment options after sorafenib failure in patients with hepatocellular carcinoma. Clin Mol Hepatol 2017;23:273-279.

5. Bruix J, Qin S, Merle P, Granito A, Huang YH, Bodoky G, et al. Regorafenib for patients with hepatocellular carcinoma who progressed on sorafenib treatment (RESORCE): a randomised, double-blind, placebo-controlled, phase 3 trial. Lancet 2017;389:56-66.

6. El-Khoueiry AB, Sangro B, Yau T, Crocenzi TS, Kudo M, Hsu C, et al. Nivolumab in patients with advanced hepatocellular carcinoma (CheckMate 040): an open-label, non-comparative, phase 1/2 dose escalation and expansion trial. Lancet 2017;389:2492-2502.

7. Abou-Alfa GK, Meyer T, Cheng AL, El-Khoueiry AB, Rimassa L, Ryoo $B Y$, et al. Cabozantinib in patients with advanced and progressing hepatocellular carcinoma. N Engl J Med 2018;379:54-63.

8. Zhu AX, Kang YK, Yen CJ, Finn RS, Galle PR, Llovet JM, et al. Ramucirumab after sorafenib in patients with advanced hepatocellular carcinoma and increased alpha-fetoprotein concentrations (REACH-2): a randomised, double-blind, placebo-controlled, phase 3 trial. Lancet Oncol 2019;20:282-296.

9. Finn RS, Merle P, Granito A, Huang YH, Bodoky G, Pracht M, et al. 
Cheol-Hyung Lee, et al. Nivolumab versus regorafenib for advanced HCC

Outcomes of sequential treatment with sorafenib followed by regorafenib for HCC: additional analyses from the phase III RESORCE trial. J Hepatol 2018;69:353-358.

10. Yau T, Hsu C, Kim TY, Choo SP, Kang YK, Hou MM, et al. Nivolumab in advanced hepatocellular carcinoma: sorafenib-experienced Asian cohort analysis. J Hepatol 2019;71:543-552.

11. Feng D, Hui X, Shi-Chun L, Yan-Hua B, Li C, Xiao-Hui L, et al. Initial experience of anti-PD1 therapy with nivolumab in advanced hepatocellular carcinoma. Oncotarget 2017;8:96649-96655.

12. Finkelmeier F, Czauderna C, Perkhofer L, Ettrich TJ, Trojan J, Weinmann $A$, et al. Feasibility and safety of nivolumab in advanced hepatocellular carcinoma: real-life experience from three German centers. J Cancer Res Clin Oncol 2019;145:253-259.

13. Ogasawara S, Ooka Y, Itokawa N, Inoue M, Okabe S, Seki A, et al. Sequential therapy with sorafenib and regorafenib for advanced hepatocellular carcinoma: a multicenter retrospective study in Japan. Invest New Drugs 2020;38:172-180.

14. Scheiner B, Kirstein MM, Hucke F, Finkelmeier F, Schulze $K$, von Felden J, et al. Programmed cell death protein-1 (PD-1)-targeted immunotherapy in advanced hepatocellular carcinoma: efficacy and safety data from an international multicentre real-world cohort. Aliment Pharmacol Ther 2019;49:1323-1333.

15. Yoo C, Park JW, Kim YJ, Kim DY, Yu SJ, Lim TS, et al. Multicenter retrospective analysis of the safety and efficacy of regorafenib after progression on sorafenib in Korean patients with hepatocellular carcinoma. Invest New Drugs 2019;37:567-572.

16. Dip Borunda AK, Campos Gomez S, Huitzil Melendez FD. Cause of death in patients with hepatocellular carcinoma ( $\mathrm{HCC}$ ) according to Barcelona Clinic Liver Cancer System (BCLC): Tumor versus nontumor-related mortality. J Clin Oncol 2011;29(15_suppl):e14594.

17. Giannini EG, Farinati F, Ciccarese F, Pecorelli A, Rapaccini GL, Di Marco $M$, et al. Prognosis of untreated hepatocellular carcinoma. Hepatology 2015;61:184-190.

18. Shiba S, Kondo S, Sakamoto Y, Sasaki M, Koga F, Hosoi H, et al. Outcome and cause of death of hepatocellular carcinoma patients with extrahepatic metastases. J Clin Oncol 2015;33:456.

19. Heimbach JK, Kulik LM, Finn RS, Sirlin CB, Abecassis MM, Roberts $L R$, et al. AASLD guidelines for the treatment of hepatocellular carcinoma. Hepatology 2018;67:358-380.

20. Choi J, Han S, Kim N, Lim YS. Increasing burden of liver cancer despite extensive use of antiviral agents in a hepatitis $B$ virus-endemic population. Hepatology 2017;66:1454-1463.

21. Lee JH, Cho Y, Kim HY, Cho EJ, Lee DH, Yu SJ, et al. Serum tumor markers provide refined prognostication in selecting liver transplantation candidate for hepatocellular carcinoma patients beyond the Milan criteria. Ann Surg 2016;263:842-850.

22. Lencioni R, Llovet JM. Modified RECIST (mRECIST) assessment for hepatocellular carcinoma. Semin Liver Dis 2010;30:52-60.

23. Curtis LH, Hammill BG, Eisenstein EL, Kramer JM, Anstrom KJ. Using inverse probability-weighted estimators in comparative effectiveness analyses with observational databases. Med Care 2007;45(10 Suppl 2):S103-S107.

24. Rosenbaum PR, Rubin DB. The central role of the propensity score in observational studies for causal effects. Biometrika 1983;70:41-55.

25. Kambhampati S, Bauer KE, Bracci PM, Keenan BP, Behr SC, Gordan $J D$, et al. Nivolumab in patients with advanced hepatocellular carcinoma and Child-Pugh class B cirrhosis: safety and clinical outcomes in a retrospective case series. Cancer 2019;125:3234-3241.

26. Ikeda M, Sung MW, Kudo $M$, Kobayashi $M$, Baron AD, Finn RS, et al. A phase $1 \mathrm{~b}$ trial of lenvatinib (LEN) plus pembrolizumab (PEM) in patients (pts) with unresectable hepatocellular carcinoma (uHCC). J Clin Oncol 2018;36(15_suppl):4076.

27. Pishvaian MJ, Lee MS, Ryoo BY, Stein S, Lee KH, Verret W, et al. LBA26 updated safety and clinical activity results from a phase lb study of atezolizumab + bevacizumab in hepatocellular carcinoma (HCC). Ann Oncol 2018;29(Suppl 8):viii718-viii719.

28. Uchikawa S, Kawaoka T, Aikata H, Kodama K, Nishida Y, Inagaki Y, et al. Clinical outcomes of sorafenib treatment failure for advanced hepatocellular carcinoma and candidates for regorafenib treatment in real-world practice. Hepatol Res 2018;48:814-820.

29. Lee JS, Kim BK, Kim SU, Park JY, Ahn SH, Seong JS, et al. A survey on transarterial chemoembolization refractoriness and a real-world treatment pattern for hepatocellular carcinoma in Korea. Clin Mol Hepatol 2020;26:24-32.

30. Lee HW, Kim HS, Kim SU, Kim DY, Kim BK, Park JY, et al. Survival estimates after stopping sorafenib in patients with hepatocellular carcinoma: NEXT score development and validation. Gut Liver 2017;11:693-701.

31. Rao N, Rizk EM, Hwang R, Gartrell RD, Makkar J, Oza J, et al. Nivolumab for hepatocellular carcinoma (HCC) in a real-world context. J Clin Oncol 2019;37(15_suppl):e15675.

32. Borcoman E, Nandikolla A, Long G, Goel S, Tourneau CL. Patterns of response and progression to immunotherapy. Am Soc Clin Oncol Educ Book 2018;38:169-178.

33. Grierson P, Crites D, Ruzinova MB, Yano M, Lim KH. Distinct clinical and magnetic resonance features of metastatic hepatocellular carcinoma treated with pembrolizumab: a case report of late response after pseudoprogression. Hepatol Commun 2017;2:148-151.

34. Mamdani H, Wu H, O'Neil BH, Sehdev A. Excellent response to AntiPD-1 therapy in a patient with hepatocellular carcinoma: case report and review of literature. Discov Med 2017;23:331-336.

35. Seymour L, Bogaerts J, Perrone A, Ford R, Schwartz LH, Mandrekar S, et al. iRECIST: guidelines for response criteria for use in trials testing immunotherapeutics. Lancet Oncol 2017;18:e143-e152. 